논문 2013-08-33

\title{
Daisy Chain Method for Control Allocation Based Fault-Tolerant Control
}

\author{
Jiyeon Kim, Inseok Yang, Dongik Lee*
}

\begin{abstract}
This paper addresses a control allocation method for fault-tolerant control by redistributing redundant control surfaces. The proposed method is based on a classical daisy chain approach for the compensation of faulty actuators. The existing daisy chain method calculates a desired moment according to a number of actuator groups. However, this method has a significant limitation; that is, any faulty actuator belonging to the last actuator group cannot be compensated, since there is no more redundant actuator group that can be used to generate the required moments. In this paper, a modified daisy chain method is proposed to overcome this problem. Using the proposed method, the order of actuator groups is readjusted so that actuator groups containing any faulty actuator are always placed in an upper group instead of the last one. A set of simulation results with an F-18 HARV aircraft demonstrate that the proposed method can achieve better performance than the existing daisy chain method.
\end{abstract}

Keywords : Fault-tolerant control, Control allocation, Daisy chain method, Aerial vehicle systems

\section{Introduction}

Recent research into modern aircraft design stresses the use of redundant control surfaces for improving the performance of fault-tolerant control (FTC). The fundamental purpose of the FTC scheme is to maintain stability and certain performance requirement of the overall system despite of the actuator or sensor faults. As one of the most commonly employed FTC method, the control allocation (CA) method has been proven to be useful in *Corresponding Author (dilee@ee.knu.ac.kr) Received: 11 Mar. 2013, Revised: 6 May 2013, Accepted: 14 May 2013.

J. Kim, D. Lee: Kyungpook National University

I. Yang: Center for IT \& Automobile Convergence

* This research was supported by the MSIP(Ministry of Science, ICT \& Future Planning), Korea, under the C-ITRC(Convergence Information Technology Research Center) support program (NIPA-2013-H0401-13-1005) supervised by the NIPA(National IT Industry Promotion Agency.)

c J. IEMEK 2013 Oct.: 8(5) 265-272

ISSN : 1975-5066

http://dx.doi.org/10.14372/IEMEK.2013.8.5.265 restoring system safety and performance by using redundant actuators to replace faulty actuators, especially in an over-actuated system. Using the CA method, acceptable performance can be achieved without changing the complex flight controller in the presence of actuator failures [1]. Consequently, the CA problem has been extensively studied, including direct allocation [2], pseudo-inverse [3], daisy chain [4, 5], non-linear programming [6], and approximate quadratic programming [7]. These methods are considered as a static optimization problem for CA.

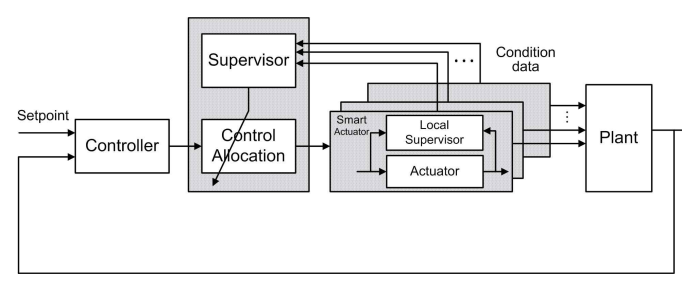

Fig. 1 Fault-tolerant control system with smart actuators 
In this paper, a modified daisy chain method is presented to overcome the inherent drawbacks of the existing daisy chain method $[4,5]$. In the existing daisy chain method, the input vector is divided into several groups in order to achieve the desired moments. In this case, all of the actuator groups are calculated in order. Therefore, any actuator failure belonging to the last group cannot be compensated as no more actuator group is available to replace the faulty one. The proposed daisy chain method can solve this problem by reordering the actuator groups so that those groups with faulty actuators are placed into the upper groups.

As shown in Fig. 1, the proposed method utilizes smart actuators [8]. A smart actuator is an actuating system that can offer local diagnosis, local compensation, and $\mathrm{bi}^{-}$ directional communications by using the built-in processor and fieldbus network [9]. In this paper, a set of condition data generated by each actuator to describe its health status is used by the supervisor in order to manage the control allocation module. It is assumed that actuator failures are locally detected within each smart actuator using a selfdiagnostic function. Using these condition data, including the information on actuator failure, the supervisor reconfigures the CA module in order to compensate degraded system performance due to faulty actuators.

\section{Equations and theorems}

\section{Control allocation method}

Control allocation is a method that involves distributing a desired control input into the set of redundant actuators. Consider the linearized dynamics equation given by

$$
\begin{aligned}
& x=x+B u \\
& y=C x
\end{aligned}
$$

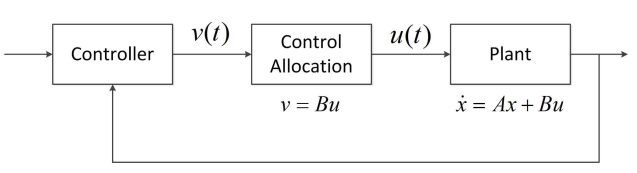

Fig. 2. Control allocation in a typical system

where $x \in R$ is the state vector, $u \in R^{m}$ is the control input vector with constraints such as $\left.u_{\text {min }}, u_{\max }\right]$, and $y \in R^{q}$ denotes the system outputs. $A \in R^{n \times n}, B \in R^{n \times m}$, and $C \in R^{q \times n}$ are the state matrix, the control effectiveness matrix, and the output matrix, respectively. Given a desired input $v \in R^{n}$, the control allocation problem involves finding the control input $u$ such that the following conditions are satisfied:

$$
\begin{gathered}
B u=v \\
\text { subjects to } u_{\min } \leq u \leq u_{\max }
\end{gathered}
$$

In other words, as shown in Fig. 2, the control allocation method calculates the optimal actuator input $\mathrm{u}$ that satisfies equation (2) to generate the virtual input $v$, which is determined by the controller.

\section{Daisy chain method}

The daisy chain method, which is a control allocation technique with redundant groups of actuators, was introduced by Buffington and Enns [4]. The key idea is that the actuator input $u$ and the matrix $B$ are allocated into $M$ groups as follows:

$$
\begin{aligned}
& u=\left[\begin{array}{llll}
U_{1}: U_{2}: & \cdots & : & U
\end{array}\right]^{T}, \\
& B=\left[\begin{array}{llll}
B_{1}: B_{2}: & \cdots & B_{M}
\end{array}\right] .
\end{aligned}
$$

Then, the control allocation problem of equation (2) can be represented as solving

$$
\begin{aligned}
B u & =\left[B_{1}: B_{2}: \cdots: B_{M}\right]\left[U_{1}: U_{2}: \cdots: U_{M}\right]^{T} \\
& =B_{1} U_{1}+B_{2} U_{2}+\cdots+B_{M} U_{M}=v .
\end{aligned}
$$

As shown in Fig. 3, the daisy chain method usually divides the actuator inputs into two groups, 


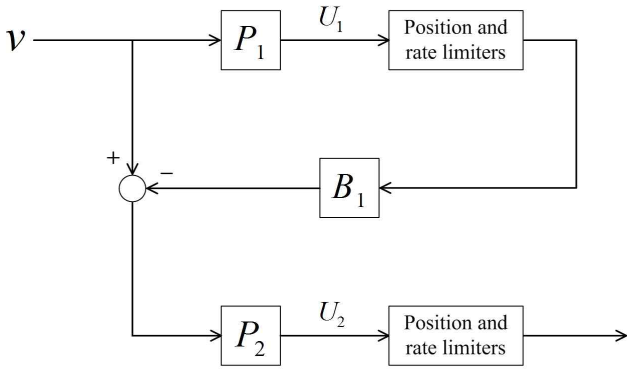

Fig. 3 Structure of daisy chain problem in case of two groups [10]

i.e., 2 [10]. Firstly, the first group $U$ is allocated using the pseudo-inverse of $B_{1}$ as follows:

$$
\left.U_{1}=s a t \quad P_{1} v\right)
$$

where $P_{1}$ is the right inverse of $B_{1}$ and $\left.s a t_{U_{1}} \cdot\right)$ is the actuator saturation of the first actuator group. If the virtual input is not satisfied by the first group, then the second group can be calculated to satisfy the remaining demands as follows:

$$
U_{2}=\operatorname{sat}_{U_{2}}\left(P_{2}\left(v-B_{1} U_{1}\right)\right) .
$$

If the actuators belong to more than two groups, this procedure is finished when the virtual input is satisfied or all of the groups have been employed. The procedure of the daisy chain method can be summarized as

$$
\begin{aligned}
& U_{1}=\operatorname{sat}_{U_{1}}\left(P_{1} v\right) \\
& U_{2}=\operatorname{sat}_{U_{2}}\left(P_{2}\left(v-B_{1} U_{1}\right)\right) \\
& \vdots \\
& \left.U_{M}=\operatorname{sat}_{U} P_{M}\left(v-{ }_{k=1}^{M-1} B_{k} U_{k}\right)\right) .
\end{aligned}
$$

State equation (1), which is applied to the daisy chain method can be represented as

$$
\dot{x}=A x+B_{1} U_{1}+B_{2} U_{2}+\cdots+B_{M} U_{M}
$$

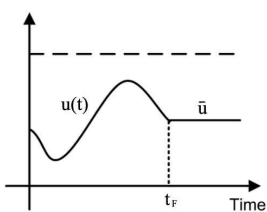

(a) Lock-in-Place

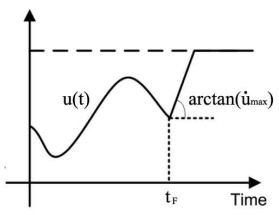

(c) Hard-over

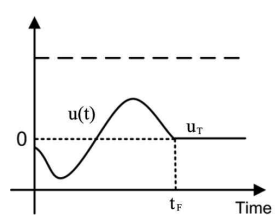

(b) Float

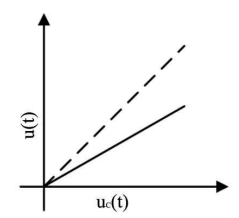

(d) Loss of Effectiveness
Fig. 4 Types of actuator failure [11]

\section{Modeling of actuator failures}

As shown in Fig. 4, typical actuator failures in flight control include lock-in-place (LIP), float, hard-over failure, and loss-ofeffectiveness (LOE) [11]. These actuator failures can be divided into two categories: (a) total loss of effectiveness and (b) partial loss of effectiveness [12]. In the case of the first category, the effectiveness of the actuator becomes zero. LIP, float, and hard-over failures are included in the total failure category [13]. On the other hand, LOE is an example of the second category, partial loss of effectiveness, which is characterized by reduced actuator gain with respect to its nominal value.

The dynamics of the $i$-th faulty actuator can be written as [8]

$$
u_{i}=\left(1-\alpha_{i}\right) \gamma_{i} \delta_{i}+\alpha_{i} \delta_{i}
$$

where $\delta_{i}$ and $\bar{\delta}_{i}$ are the ideal actuator position and actuator response with a total failure of the $i$-th actuator, respectively. The condition data $\alpha_{i}$ and $\gamma_{i}$ are provided by the actuator itself. The switching variable $\alpha_{i}$ satisfies the following:

$$
\alpha_{i}=0 \text {, normal or LOE failure }
$$


The performance index , which is a quantitative value in the range of $[0,1]$ can be defined as

$$
\gamma_{i}=\left\{\begin{array}{cc}
0, & \text { total failure } \\
\varepsilon(0<\varepsilon<1), & \text { LOE failure } \\
1, & \text { normal }
\end{array}\right.
$$

In this paper, actuator modeling for the proposed method considers both LOE and total failures. The presence of fault with the $i^{- \text {th }}$ actuator can be determined by using the condition data, such as $\alpha_{i}$ and $\gamma_{i}$.

In the case of total failure, the actuator output is independent of the input. Hence, this failure can be considered as follows:

$$
\left.{ }_{j} U_{j}=B_{j} I-\Omega_{j}\right) U_{j}+B_{j} \Omega_{j} U_{j}
$$

where $\Omega_{j}$ is a diagonal matrix of $\alpha$ in the $j$-th group and $\bar{U}_{j}$ is the matrix of responses of actuators belonging to the $j$-th group.

If an LOE failure occurs in the $i$-th actuator, which belongs to the $j$-th group, then the actuator performance is degraded to the performance index $\gamma$, which ranges from $[0$, 1]. Therefore, LOE failure in the $j$-th group can be compensated by adding the effectiveness matrix into the matrix gain $P_{j}$ as follows:

$$
P_{j}^{*}=\left\{B_{j} \Gamma_{j}\left(I-\Omega_{j}\right)+B_{j} \Omega_{j}\right\}^{\dagger}
$$

where $\Gamma_{j}$ is a diagonal matrix of $\gamma$ in the $j$-th actuator group and $\cdot)^{\dagger}$ is the pseudo inverse of $(\cdot)$. Therefore, the general equation of the daisy chain method (7) can be represented as follows:

$$
\begin{aligned}
U_{j} & \left.=\text { sat } P_{j}^{*}\left(v-\sum_{k=1}^{j-1} B_{k} U_{k}^{*}\right)\right) \\
& \left.=\operatorname{sat}_{U_{j}}\left(P_{j}^{*}\left(v-\sum_{k=1}^{j-1} B_{k}\left(I-\Omega_{k}\right) U_{k}+B_{k} \Omega_{k} U_{k}\right)\right)\right)
\end{aligned}
$$

\section{The proposed daisy chain method}

In contrast to the case of LOE failure, when the last group develops total failure, the effect of the faulty actuator cannot be compensated since there is no group that can compensate the lost effect due to the failure. The proposed solution to overcome this problem is to prioritize actuator groups according to the existence of any total failure. That is, actuator groups containing any total failure are calculated in order to solve the control allocation problem first, after which the remaining demands are calculated by the next groups within their actuator limits.

The switching variable $f_{j}$ to classify the groups, whether or not they contain any total failure, is defined as

$$
f_{j}=\begin{array}{ll}
0, & \text { if } \Omega_{j}=O \\
1, & \text { if } \Omega_{j} \neq O
\end{array}
$$

If the condition variable $\alpha_{i}$ of the $i$-th actuator from the $j$-th group is equal to 1 due to total failure, then the variable $f_{j}$ becomes 1 . The expression to compensate the effect of the actuator group with total failure is given by

$$
F_{j}=\operatorname{sat}_{F_{j}}\left(f_{j} P_{j}^{*}\left(v-\sum_{k=1}^{j-1}\left(B_{k}\left(I-\Omega_{k}\right) F_{k}+B_{k} \Omega_{k} \overline{U_{k}}\right)\right)\right)
$$

Actuator groups without any total failure, having a null matrix of the switching variable, can be calculated as

$N_{j}=s a t_{N_{j}}$
$\left(\left(1-f_{j}\right) P_{j}^{*}\left(v-\sum_{k=1}^{M}\left(B_{k}\left(I-\Omega_{k}\right) F_{k}+B_{k} \Omega_{k} \overline{U_{k}}\right)-\sum_{l=1}^{j-1} B_{l} N_{l}\right)\right)$.

Firstly, $F_{j}$ is calculated for all groups for $j=1, \cdots, M$. If the $j$-th actuator group is a normal actuator group or includes only LOE failure, then $F_{j}$ goes into the zero vector as $f_{j}=0$ according to (16). After calculating $F_{j}$ for all actuator groups, $N_{j}$ is also calculated for $j=1, \cdots, M$. Therefore, 
the actuator input vector of the $j$-th group can be restated as

$$
F_{j}+N_{j}
$$

\section{Computer simulation}

\section{Simulation conditions}

The proposed method is evaluated through a set of computer simulations using a linearized mathematical model of an F-18 HARV (High Alpha Research Vehicle) aircraft. Linearization is carried out under operating conditions that correspond to an altitude of $10,000 \mathrm{ft}$, speed of Mach 0.23 , and angle-of-attack of $30^{\circ}$ [14]. This paper considers a problem with eight controls and three moments. For the considered flight case, the effectiveness matrices $B_{1}$ and $B_{2}$, which are partitioned into two matrices, are given by

$$
\begin{gathered}
\left.B_{1}=\begin{array}{rrr}
{\left[2.538 e^{-2}\right.} & -3.801 e^{-1} & -1.681 e^{-3} \\
-2.538 e^{-2} & -3.801 e^{-1} & 1.681 e^{-3} \\
2.743 e^{-2} & -3.495 e^{-2} & -9.251 e^{-3} \\
-2.743 e^{-2} & -3.495 e^{-2} & 9.251 e^{-3} \\
1.922 e^{-3} & 1.681 e^{-5} & -3.827 e^{-2}
\end{array}\right], \\
B_{2}=\left[\begin{array}{ccc}
-2.500 e^{-2} & 2.500 e^{-2} & 4.500 e^{-4} \\
1.125 e^{-1} & 1.125 e^{-1} & 0.000 e+0 \\
0.000 e+0 & 0.000 e+0 & -7.500 e^{-2}
\end{array}\right] .
\end{gathered}
$$

The first actuator group $B_{1}$ contains the left and right horizontal tails, ailerons, and the combined rudders. The left and right rudders are treated as a single control in order to move together. The second actuator group $B_{2}$ contains the left and right thrust vectoring nozzles and yaw thrust vectoring. The position constraints of the controls are presented as follows:

$$
\left.u_{\min }=\begin{array}{r}
\lceil-0.419 \\
-0.419 \\
-0.436 \\
-0.436 \\
-0.524 \\
-0.524 \\
-0.524 \\
\lfloor-0.524
\end{array}\right], u_{\max }=\left[\begin{array}{c}
0.183 \\
0.183 \\
0.733 \\
0.733 \\
0.524 \\
0.524 \\
0.524 \\
0.524
\end{array}\right][\mathrm{rad}] .
$$

Three virtual moments, namely moment coefficients of pitch, roll, and yaw, are chosen arbitrarily. In this simulation, an LOE failure and LIP failure, which is an example of total failure, in the right thrust vectoring control are considered. The faulty actuators belong to the second group.

\section{Simulation results}

The simulation results in the presence of $50 \%$ LOE failure in the right thrust vectoring nozzle are depicted in Figs. 5-6. In Fig. 5, the trajectories of coefficients for roll, pitch, and yaw moments are compared between the existing daisy chain method (uncompensated) and the proposed method (compensated). In Fig. 5, performance is degraded due to LOE failure in the case of the existing daisy chain method. However, the results with compensation using the proposed method show that performance is maintained similar to the virtual moment. Further, using the proposed method, the errors in the reference virtual moment are nearly zero as shown in Fig. 6.

Figs. 7-8 show the simulation results with LIP failure, which is a total failure. There are errors in the roll and pitch moment coefficients due to LIP failure in the existing method. In contrast, the resulting performance is acceptable by using the modified daisy chain method even in the presence of LIP failure with the second actuator group.

\section{Conclusion}

This paper presented a modified daisy chain method to overcome the limitations of the existing daisy chain method. In the proposed method, by reordering the actuator groups according to the existence of failure, the difficulty in compensating any failure with the last actuator group could be overcome. The proposed method can be implemented on low-cost embedded processors due to not need a mathematical model of the plant. The performance of the proposed method has been evaluated by a set of computer simulations with an F-18 HARV aircraft. The simulation results show that the proposed method can effectively deal with both types of actuator failures, LOE and LIP, which are developed in the last actuator group. 

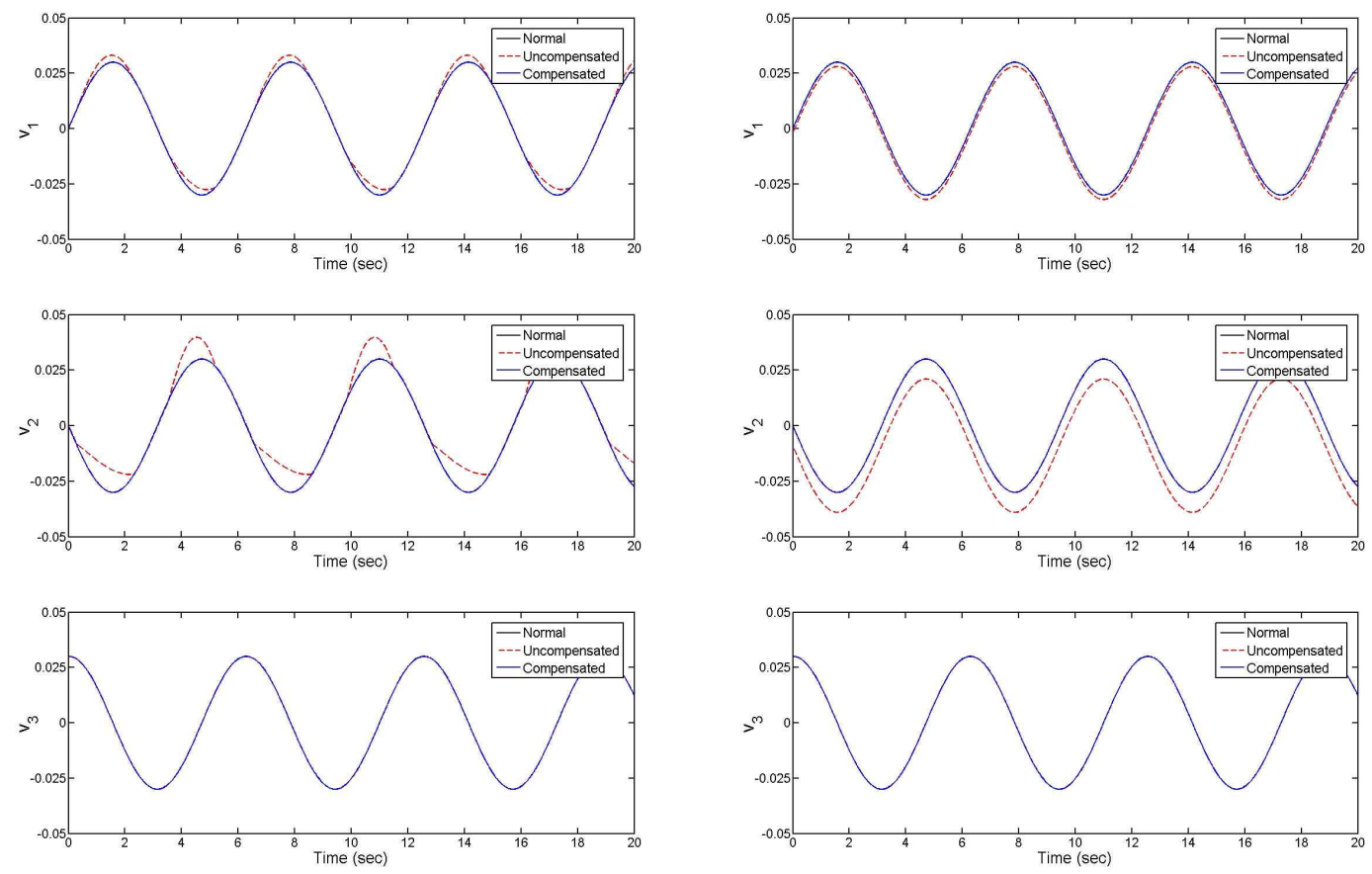

Fig. 5 Trajectories of moment coefficients with 50\% LOE failure

Fig. 7 Trajectories of moment coefficients with LIP failure
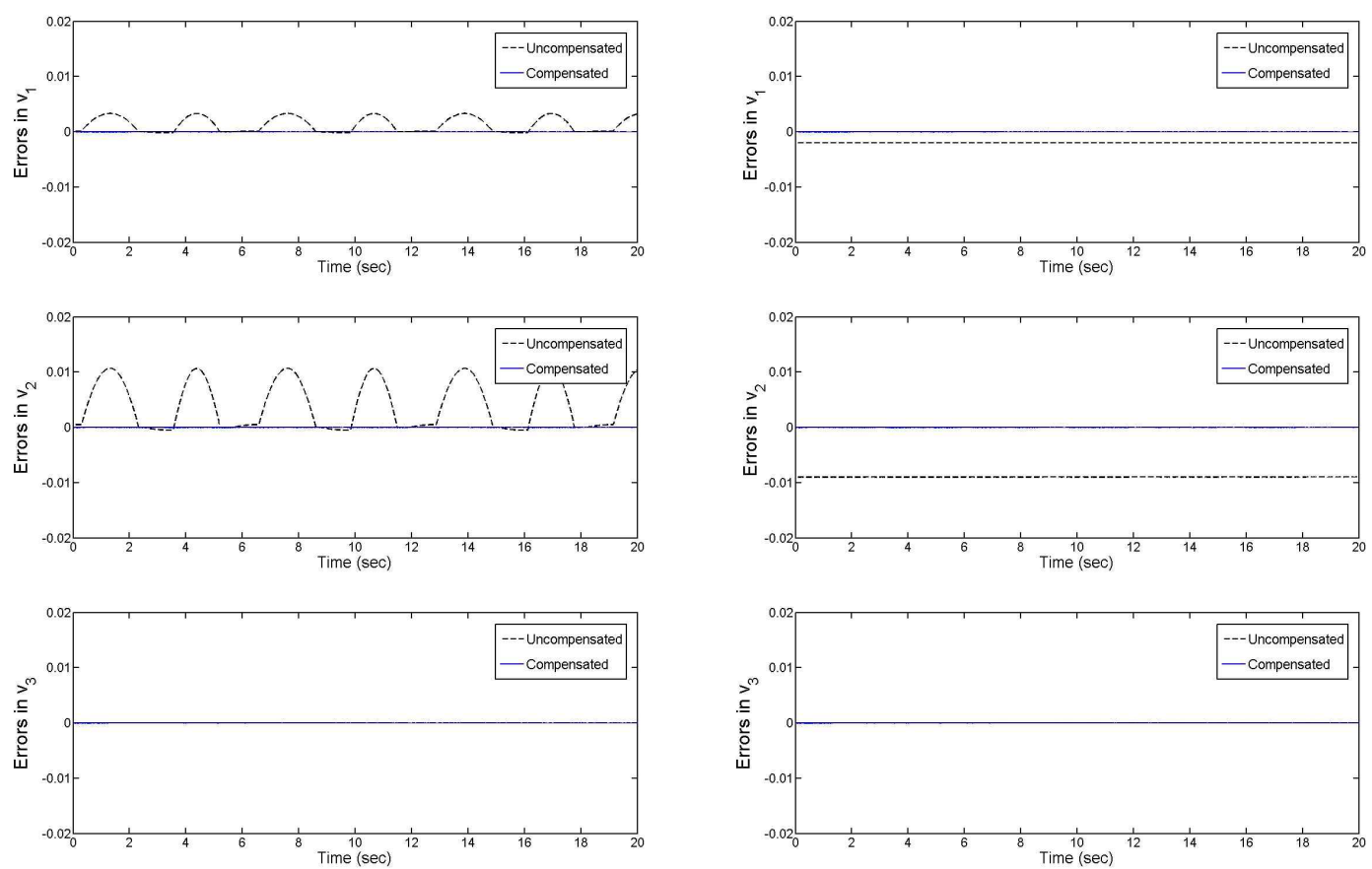

Fig. 6 Errors in moment coefficients with $50 \%$ LOE failure

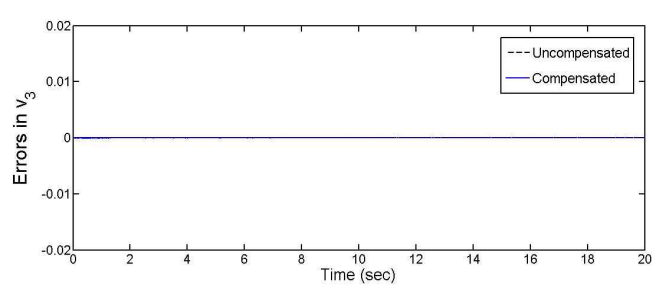

Fig. 8 Errors in moment coefficients with LIP failure 


\section{References}

[1] R. Eberhardt D. Ward, "Indirect adaptive flight control of a tailless fighter aircraft," Proceedings on AIAA Guidance, Control, and Dynamics Conference and Exhibit, 1999.

[2] W. Durham, "Constrained Control Allocation," Journal of Guidance, Control, and Dynamics, Vol. 16, No. 4, pp.717-725, 1993.

[3] J. Davison, F. Lallman, W. Bundick, "Integrated reconfigurable control allocation," Proceedings on AIAA Guidance, Navigation, and Control Conference, 2001.

[4] J. Buffington, D. Enns, "Lyapunov stability analysis of daisy chain control allocation," Journal of Guidance, Control, and Dynamics, Vol. 19, No. 6, pp.1226-1230, 1996.

[5] K.A. Bordignon, "Constrained control allocation for systems with redundant control effectors," Ph.D. Dissertation, Department of Aerospace Engineering, Virginia Polytechnic Institute and State University, USA, 1996.

[6] T.A. Johansen, T.I. Fossen, S.P. Berge, "Constrained nonlinear control allocation with singularity avoidance using sequential quadratic programming," IEEE Transactions on Control Systems Technology, Vol. 12, No. 1, pp.211-216, 2004.

[7] J.A.M. Petersen, M. Bodson, "Constrained quadratic programming techniques for control allocation," IEEE Transactions on Control Systems Technology, Vol. 14, No. 1, pp.91-98, 2006.

[8] I. Yang, D. Kim, D. Lee, "Fault-tolerant control strategy based on control allocation using smart actuators," Proceedings on Conference of Control and Fault Tolerant Systems, pp.377-381, 2010.

[9] D. Lee, "Distributed real-time fault-tolerant control using smart actuators and timetriggered communication," Ph.D. Dissertation, Department of Automatic Control and Systems Engineering, Sheffield University, UK, 2002.

[10] O. Harkegard, "Backstepping and control allocation with applications to flight control,"
Ph.D. Dissertation, Department of Electrical Engineering, Linkoping University, Sweden, 2003.

[11] E. Sobhani-Tehrani, K. Khorasani, Fault diagnosis of nonlinear systems using a hybrid approach, Springer, 2009.

[12] J.D. Boskovic, J. Redding, R.K. Mehra, "Robust fault-tolerant flight control using a new failure parameterization," Proceedings on American Control Conference, pp.5753-5758, 2007.

[13] M. Bodson, J.E. Groszkiewicz, "Multivariable adaptive algorithms for reconfigurable flight control," IEEE Transaction on Control Systems Technology, Vol. 5, No. 2, pp. 217-229, 2007.

[14] W. Durham, K.A. Bordignon, "Multiple control effector rate limiting," Journal of Guidance, Control, and Dynamics, Vol. 19, No. 1, pp.30-37, 1996. 


\section{Authors}

\section{Jiyeon Kim}

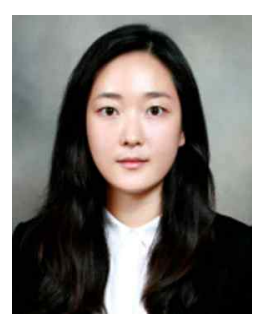

2010: B. E. degree in Electronics Eng. from Kyungpook National University, Korea 2012: M. S. degree in Electronics Eng. from Kyungpook National University, Korea

Current: Ph. D. candidate in the School of Electronics Engineering at Kyungpook National University.

Research interests: Formation control, Fault-tolerant control, Robot safety, and Fleet control

Email: jiykim@knu.ac.kr

\section{Inseok Yang}

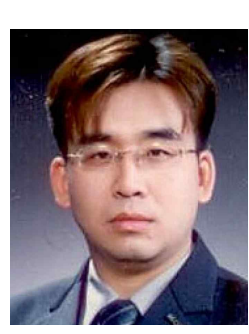

2001: B. S. degree in the department of Mathematics from Kyungpook National University, Korea 2005: M. S. degree in the dep. of Industrial Applied Mathematics

from Kyungpook National University, Korea

2011: Ph. D. degree in the dep. of Industrial Applied Mathematics from Kyungpook National University, Korea

Current: Postdoctoral researcher at the Center for IT \& Automobile Convergence.

Research interests: Flight control, Fault-tolerant control, and Networked embedded systems

Email: jewill@hanmail.net

\section{Dongik Lee}

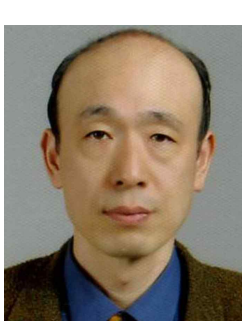

1987: B. E. degree in Electronics Eng. from Kyungpook National University, Korea 1990: M. S. degree in Electronics Eng. from Kyungpook National University, Korea

1990 1997: Researcher at Agency for Defense Development (ADD), Korea.

2002: Ph. D. in the department of Automatic Control \& Systems Engineering from Sheffield University, U. K. 2002 2005: Dependable Real Time Systems (DRTS) Ltd Co-founder and CTO, U. K.

Current: Associate professor in Kyungpook National University.

Research interests: Fault Tolerance and System Safety, Fault Diagnosis, Industrial Networks, Wind Turbines, and Intelligent Vehicles

Email: dilee@ee.knu.ac.kr 